\title{
Laplace integrals in partial differential equations in papers of Bogdan Ziemian
}

\author{
by GRZEGORZ Łysik (Warszawa)
}

\begin{abstract}
Fundamental solutions to linear partial differential equations with constant coefficients are represented in the form of Laplace type integrals.

1. Problem. Let $P$ be a polynomial in $n$ complex variables and consider the tempered distribution

$$
\widehat{E}_{0}=\operatorname{reg} \frac{1}{P(0+i \cdot)},
$$

which is the regularization of the function $\mathbb{R}^{n} \ni \beta \mapsto 1 / P(0+i \beta)$ to a tempered distribution on $\mathbb{R}^{n}$. The problem consists essentially in establishing a real Laplace inversion formula for $\widehat{E}_{0}$. Recall that it is easy to obtain an imaginary inversion formula for $\widehat{E}_{0}$ : namely one takes the inverse Fourier transform of $\widehat{E}_{0}$,
\end{abstract}

$$
E_{0}(y)=\operatorname{reg} \int_{i \mathbb{R}^{n}} \frac{e^{-y \theta}}{P(\theta)} d \theta .
$$

Then $E_{0}$ is a tempered fundamental solution of the operator $P\left(D_{y}\right)$, i.e.

$$
P\left(D_{y}\right) E_{0}=c \delta_{(0)} \text {. }
$$

It follows from the classical results of Ehrenpreis [Eh] and Palamodov $[\mathrm{P}]$ that $E_{0}$ can be represented by

$$
E_{0}(y)=\int_{\text {Char } P} e^{-y \theta} \mu(d \theta)
$$

where $\mu$ is a bounded Radon measure on the complex characteristic set of $P$,

$$
\text { Char } P=\left\{z \in \mathbb{C}^{n}: P(z)=0\right\} \text {. }
$$

2000 Mathematics Subject Classification: 35A20, 35C15.

Key words and phrases: linear partial differential equations, Laplace representations, Leray residue formula, Nilsson integrals.

The paper contains also some ideas presented by Bogdan Ziemian on his seminar. 
In the case when $P$ has no multiple factors the existence of $\mu$ follows from the Riesz theorem. Thus, in general, it is not possible to get from the representation (2) (or its distributional version) the information about the behaviour of solutions at infinity. Furthermore, the measure $\mu$ is not uniquely determined.

The aim of B. Ziemian's work [Z1] was to obtain a representation of fundamental solutions of $P$ in the form (2) which remains valid for $y \rightarrow \infty$ as well as an analytic description of the measure $\mu$. To this end he put forward the idea of replacing the methods of functional analysis by the ones of complex analysis, in particular by the Leray residue formulas. Intuitively the idea seems to be easy: starting from the representation of $E_{0}$ given by the Fourier integral (1) one completes the imaginary plane $0+i \mathbb{R}^{n}$ to a cycle (by adding points at infinity) and then deforms it in such a way that it can be considered as a bundle of spheres over some sets $\Gamma_{j} \subset$ Char $P(j=1, \ldots, p)$ diffeomorphic to the $(n-1)$-dimensional octant $\overline{\mathbb{R}}_{+}^{n-1}$. Then taking the Leray residue in a normal direction to Char $P$ one gets the heuristic formula

$$
E_{0}(y)=\sum_{j=1}^{p} \int_{\Gamma_{j}} e^{-y \theta} \operatorname{res} \frac{d \theta}{P(\theta)} .
$$

Clearly, in doing so the geometry of Char $P$ enters in a crucial way. Furthermore, the program faces serious difficulties. Firstly, the classical Leray residue theory does not apply since the cycle $0+i \mathbb{R}^{n}$ intersects the singular set of the integrand. So one needs to regularize divergent integrals and it is convenient to regard the residue form as a current (in the sense of de Rham). The second difficulty is connected with the unboundedness of the cycle; so one needs to control the growth of the integrand at infinity. Unfortunately, after computing the residue in one variable good estimates in the other variables are lost. To regain them a variant of the Phragmén-Lindelöf theorem is applied. Note also that the residue currents are to be evaluated at functions with exponential growth at infinity; we call such currents Laplace currents.

The theory presented in [Z1] is parallel to the theory of Borel summability of formal solutions to ordinary differential equations with nonregular singularities (J. Ecalle, B. Malgrange, J. Braaksma). B. Ziemian expected that it would find applications to nonlinear elliptic equations giving a geometric description of the singularities of their solutions. Indeed, the formula (3) is well adapted to nonlinear operations. The sets $\Gamma_{j}$ are $(n-1)$-dimensional and can be deformed within the $(2 n-2)$-dimensional characteristic set, which enables choosing them in such a way that the set $\Gamma_{i}+\Gamma_{j}$ still has small dimension. The observed multidimensional resurgence effect in the spirit of J. Ecalle opens the possibility to create a multidimensional version of the "alien calculus" suitable to study singularities of solutions. 
2. The main result. As mentioned above the program can be realized only under suitable assumptions on $P$. The main assumptions are the ellipticity condition $\mathbf{E}$ and condition $\mathbf{B}$ on the growth of the iterated discriminant roots of $P$. Both conditions will be described in Section 4. However, we also need a technical condition (satisfied in a generic case).

Condition A. We say that $P$ satisfies condition $\mathbf{A}$ (relative to 0 ) if there exist a finite number of points $B^{1}, \ldots, B^{k} \in \mathbb{R}$ such that

$$
\text { Char } P \cap\left(0+i \mathbb{R}^{n}\right) \subset \bigcup_{j=1}^{k}\left\{z \in \mathbb{C}^{n}: \operatorname{Re} z=0, \operatorname{Im} z_{1}=B^{j}\right\} .
$$

TheOREM. Let $P$ be a complex polynomial in $n$ variables satisfying conditions $\mathbf{A}, \mathbf{B}$ and $\mathbf{E}$. Then any tempered solution $u$ of $P\left(D_{y}\right) u=\delta_{(0)}$ can be represented by the Laplace integrals

$$
u(y)=\sum_{j \in \Sigma_{0}(P)} \sum_{\sigma \in\{+,-\}^{n-1}} T_{\sigma}^{j}[\exp \{-y z\}] \quad \text { for y large enough, }
$$

where $T_{\sigma}^{j}$ are Laplace $(n-1)$-currents supported by sets $\Gamma_{\sigma}^{j}$ diffeomorphic to $\overline{\mathbb{R}}_{+}^{n-1}$ and contained in Char $P \cap\{z: \operatorname{Re} z \geq 0\}$ with vertices in the set $\Sigma_{0}(P)$ (see Section 4). For each $j \in \Sigma_{0}(P)$ and $\sigma \in\{+,-\}^{n-1}$ the Laplace current $T_{\sigma}^{j}$ can be so chosen that restricted to Int $\Gamma_{\sigma}^{j}$ it coincides with a suitable branch of the Leray residue form $\operatorname{res}(d \theta / P(\theta))$ defined on the regular part of Char $P$.

3. Leray residue form. Roughly, the Leray residue formula ([L], $[\mathrm{N}])$ can be regarded as a parameter version of the Cauchy residue formula for holomorphic functions of one variable. To be more precise take a regular complex hypersurface $S$ contained in an open set $U \subset \mathbb{C}^{n}$ and an $n$-form $\omega$ on $U \backslash S(\omega$ may be singular on $S)$. Then we can define an $(n-1)$-form res $\omega$ on $S$ by

$$
\int_{\widetilde{\gamma}} \omega=\int_{\gamma} \operatorname{res} \omega
$$

where $\gamma$ is an $(n-1)$-cycle on $S$ and $\widetilde{\gamma}$ is an $n$-cycle in $U \backslash S$ homotopic to a bundle of spheres over $\gamma$. Locally, if $S$ is given by the zero set of a holomorphic function $s$ on $U$, i.e. $S=\{z \in U: s(z)=0\}$, then

$$
\operatorname{res} \omega=\left.\frac{s \omega}{d s}\right|_{S}
$$

which is a generalization of the classical Cauchy formula. The basic feature of the Leray residue form is that it is a uniquely defined global $(n-1)$-form on $S$. 
4. Iterated discriminants and ellipticity. Recall that the (reduced) discriminant $\Delta Q$ of a polynomial $Q(\zeta)=a_{m}\left(\zeta-c_{1}\right) \ldots\left(\zeta-c_{m}\right)$ is defined by

$$
\Delta Q=a_{m}^{2 m-1} \prod_{j<k}\left(c_{j}-c_{k}\right)^{2},
$$

where in the case when $Q$ contains multiple factors we neglect them in the product.

In our case we deal with a polynomial $P$ in $n$ variables. So fixing $z_{1}$ and $\theta^{\prime \prime}=\left(\theta_{3}, \ldots, \theta_{n}\right) \in \mathbb{C}^{n-2}$ we can compute the discriminant $\Delta_{2}(P)\left(z_{1}, \theta^{\prime \prime}\right)$ of $P$ with respect to the second variable. Since $\Delta_{2}(P)$ is still a polynomial we can compute its discriminant $\Delta_{3,2}(P)$ with respect to $\theta_{3}$. Continuing the procedure we arrive at polynomials $\Delta_{k, \ldots, 2}(P)\left(z_{1}, \theta^{(k)}\right)$ for $k=2, \ldots, n\left(\theta^{(k)}=\right.$ $\left(\theta_{k+1}, \ldots, \theta_{n}\right)$ for $\left.k=2, \ldots, n-1, \theta^{(n)}=\emptyset\right)$ which are called the (reduced) iterated discriminants of $P$. Denote by $c^{2}\left(z_{1}, \theta^{\prime \prime}\right), c^{3}\left(z_{1}, \theta^{(3)}\right), \ldots, c^{n}\left(z_{1}\right)$ the multivalued roots of the iterated discriminants.

Condition B. The iterated discriminant roots $c^{k}\left(z_{1}, \theta^{(k)}\right), k=2, \ldots, n$, of $P$ grow at most linearly, i.e.

$$
\left|c^{k}\left(z_{1}, \theta^{(k)}\right)\right| \leq C\left\|\left(z_{1}, \theta^{(k)}\right)\right\| \quad \text { for }\left\|\left(z_{1}, \theta^{(k)}\right)\right\| \text { large enough. }
$$

Consider the multivalued mapping $E=E\left(z_{1}, \theta^{\prime \prime}\right)$ defined by

$$
\begin{aligned}
\left(z_{1}, \theta^{\prime \prime}\right) & \mapsto\left(z_{1}, c^{2}\left(z_{1}, c^{3}\left(z_{1}, \ldots, c^{n-1}\left(z_{1},\right.\right.\right.\right. \\
& \left.\left.\left.\left.\left.c^{n}\left(z_{1}\right)+\theta_{n}\right)+\theta_{n-1}\right), \ldots,\right)+\theta_{3}\right), \ldots, c^{n}\left(z_{1}\right)+\theta_{n}\right) .
\end{aligned}
$$

The ellipticity condition means that the mapping $E$ is positive definite in the following sense.

Condition E. For any fixed branch $\widetilde{E}$ of $E$ there exist $v=\left(v_{1}, v^{\prime \prime}\right) \in$ $\mathbb{C}^{n-1}$ with $\operatorname{Re} v \geq 0, \stackrel{\circ}{E} \in \mathbb{R}^{n}$ and $\kappa>0$ such that for any $\varepsilon \in \mathbb{R}_{+}^{n}$,

$$
\sum y_{j}\left(\stackrel{\circ}{E}^{j}+\operatorname{Re} \widetilde{E}^{j}\left(z_{1}, \theta^{\prime \prime}\right)\right) \geq c_{\varepsilon}\left\|\left(z_{1}, \theta^{\prime \prime}\right)\right\|^{\kappa}
$$

for $\mathbb{R}_{+}^{n} \ni y, y \geq \varepsilon$ and $\left\|\left(z_{1}, \theta^{\prime \prime}\right)\right\|$ large, with $\left(z_{1}, \theta^{\prime \prime}\right) \in v \mathbb{R}_{+}^{n-1}$.

Note that the $(n-1)$ th iterated discriminant roots are functions of $z_{1}$ only. Therefore, the following definition makes sense.

Definition. Suppose that $P$ satisfies Condition A relative to 0 . The vertex set $\Sigma_{0}(P)$ of $P$ is given by

$$
\Sigma_{0}(P)=\Sigma_{0}^{0}(P) \cup \Sigma_{0}^{+}(P)
$$


where

$$
\begin{array}{ll}
\Sigma_{0}^{0}=\bigcup_{j=0}^{k} i B^{j} \times \widetilde{\Sigma}_{i B^{j}}(P) & \text { (the boundary vertex set) }, \\
\Sigma_{0}^{+}=\bigcup_{\left\{z_{1}: \Delta_{n, \ldots, 2} P\left(z_{1}\right)=0, \operatorname{Re} z_{1} \geq 0\right\}} z_{1} \times \widetilde{\Sigma}_{z_{1}}(P) \quad \text { (the inner vertex set) },
\end{array}
$$

with the partial vertex set $\widetilde{\Sigma}_{z_{1}}(P)$ given by

$$
\begin{aligned}
\widetilde{\Sigma}_{z_{1}}(P)=\left\{\theta^{\prime} \in \mathbb{C}^{n-1}: \theta_{2}\right. & =c^{2}\left(z_{1}, c^{3}\left(z_{1}, \ldots, c^{n-1}\left(z_{1}, c^{n}\left(z_{1}\right)\right) \ldots\right)\right), \\
\theta_{3} & \left.\left.=c^{3}\left(z_{1}, \ldots, c^{n-1}\left(z_{1}, c^{n}\left(z_{1}\right)\right) \ldots\right)\right), \ldots, \theta_{n}=c^{n}\left(z_{1}\right)\right\} .
\end{aligned}
$$

Finally, the sets $\Gamma_{\sigma}^{j}$ for $j \in \Sigma_{0}(P), \sigma \in\{+,-\}^{n-1}$ are defined as the images of $\left(j_{1}+\overline{\mathbb{R}}_{+}\right) \times \overline{\mathbb{R}}_{+}^{n-2}$ under the branch $E_{\sigma}$ of $E$.

5. Sketch of the proof. The proof of the Theorem consists of two basic steps.

1. The study of Nilsson type integrals. For simplicity assume that Char $P \cap i \mathbb{R}^{n} \subset\left\{\operatorname{Im} z_{1}=0\right\}$. Take a cut-off function of the form $k(y)=$ $\chi\left(y_{1}\right) k^{\prime}\left(y^{\prime}\right)$ with $k^{\prime} \in C_{0}^{\infty}\left(\mathbb{R}^{n-1}\right)$ and $\chi \in C^{\infty}\left(\mathbb{R}_{+}\right)$equal to one for $y_{1}$ large and zero for $y_{1}$ small. Denote by $\mathcal{L}$ the Laplace transformation. Since $\mathcal{L} u(z)=\operatorname{reg} 1 / P(z)$ we have

$$
\mathcal{L}(k u)(z)=\int_{i \mathbb{R}^{n}} \frac{\mathcal{L} k(z-\theta)}{P(\theta)} d \theta \quad \text { for } \operatorname{Re} z_{1}<0, z^{\prime} \in \mathbb{C}^{n-1} .
$$

Next since

$$
\mathcal{L} k(z)=G\left(z_{1}\right) K^{\prime}\left(z^{\prime}\right) \quad \text { with } \quad G\left(z_{1}\right)=\frac{1}{z_{1}}+\widetilde{G}\left(z_{1}\right)
$$

where $K^{\prime}$ and $\widetilde{G}$ are entire functions, we get a Cauchy type integral with respect to $z_{1}$,

$$
\mathcal{L}(k u)(z)=\int_{i \mathbb{R}} G\left(z_{1}-\theta_{1}\right) I\left(\theta_{1}, z^{\prime}\right) d \theta_{1} \quad \text { for } \operatorname{Re} z_{1}<0,
$$

where

$$
I\left(z_{1}, z^{\prime}\right)=\int_{i \mathbb{R}^{n-1}} \frac{K^{\prime}\left(z^{\prime}-\theta^{\prime}\right)}{P\left(z_{1}, \theta^{\prime}\right)} d \theta^{\prime}
$$

is an iterated Nilsson type integral (cf. [N], [NZ], [Z1]) along the unbounded cycle $i \mathbb{R}^{n-1}$. We are interested in a holomorphic extension of $I$ and $\mathcal{L}(k u)$ to larger sets. To this end we observe that due to good growth properties of $K^{\prime}$ (it rapidly decreases along imaginary planes) the function $I_{2}\left(z_{1}, \theta^{\prime \prime}\right)$ 
defined in a neighbourhood of a fixed $\stackrel{z}{z}$ with $\operatorname{Im} \stackrel{2}{z} \neq 0$ by

$$
I_{2}\left(z_{1}, \theta^{\prime \prime}\right)=\int_{i \mathbb{R}} \frac{K^{\prime}\left(z_{2}-\theta_{2}, z^{\prime \prime}-\theta^{\prime \prime}\right)}{P\left(z_{1}, \theta_{2}, \theta^{\prime \prime}\right)} d \theta_{2}
$$

extends to a multivalued function outside the set $\left\{\left(z_{1}, \theta^{\prime \prime}\right): \Delta_{2}(P)\left(z_{1}, \theta^{\prime \prime}\right)\right.$ $=0\}$. Unfortunately, $I_{2}$ does not satisfy good estimates and so we cannot immediately continue the procedure for the remaining variables. To regain good estimates a version of the Phragmén-Lindelöf theorem stated below is applied. Finally, holomorphic extension of $\mathcal{L}(k u)$ is derived by applying properties of the modified Cauchy transformation. The Laplace currents $T_{\sigma}^{j}$ are obtained as the boundary values of $\mathcal{L}(k u)$.

2. The Phragmén-Lindelöf type theorem

Theorem ([Z2], Theorem 6.6). If $F \in \mathcal{O}(\mathbb{C})$ and there exist $0<r<t$, $\theta>0$ and $s \in \mathbb{R}$ such that for any $\varepsilon>0$,

$$
|F(\alpha+i \beta)| \leq \begin{cases}C_{\varepsilon}(1+|\beta|)^{s}\left(t e^{\varepsilon}\right)^{-\alpha} & \text { for } \alpha \leq 0, \\ C_{\varepsilon} e^{(\theta+\varepsilon)|\beta|}\left(r e^{-\varepsilon}\right)^{-\alpha} & \text { for } \alpha \geq 0,\end{cases}
$$

then there exists $\tilde{s} \in \mathbb{R}$ (one can take $\widetilde{s}=s+3$ ) such that for any $\varepsilon>0$,

$$
|F(\alpha+i \beta)| \leq \begin{cases}C_{\varepsilon}(1+|\beta|)^{\tilde{s}}\left(t e^{\varepsilon}\right)^{-\alpha} & \text { for } \alpha \leq 0 \\ C_{\varepsilon}(1+|\beta|)^{\tilde{s}}\left(r e^{-\varepsilon}\right)^{-\alpha} & \text { for } \alpha \geq 0 .\end{cases}
$$

\section{Examples}

EXAmple 1. Let $P\left(z_{1}, z_{2}\right)=z_{1}^{2}+z_{2}^{2}$ and let $\stackrel{\circ}{\alpha}=\left(\stackrel{\circ}{\alpha}_{1}, \stackrel{\circ}{\alpha}_{2}\right) \in \mathbb{R}^{2} \backslash\{0\}$. Consider $E_{\alpha} \in S_{\alpha}^{\prime}:=e^{\alpha \cdot} \cdot S^{\prime}$ given by (1) with the integral over $\stackrel{\circ}{\alpha}+i \mathbb{R}^{2}$. Then for $y, y_{2}>0$,

$$
\begin{aligned}
E_{\alpha}\left(y_{1}, y_{2}\right)= & -\frac{1}{4 \pi}\left(\int_{\Gamma_{+}} \frac{\exp \left\{-\frac{1+i}{2} y_{1} \zeta-\frac{1-i}{2} y_{2} \zeta\right\}}{\zeta} d \zeta\right. \\
& \left.+\int_{\Gamma_{-}} \frac{\exp \left\{-\frac{1-i}{2} y_{1} \zeta-\frac{1+i}{2} y_{2} \zeta\right\}}{\zeta} d \zeta\right)
\end{aligned}
$$

where $\Gamma_{ \pm}=\stackrel{\circ}{\alpha}_{1}+\stackrel{\circ}{\alpha}_{2} \pm i\left(\stackrel{\circ}{\alpha}_{2}-\stackrel{\circ}{\alpha}_{1}\right)+\overline{\mathbb{R}}_{+}$.

It is worth noting that as $\stackrel{\circ}{\alpha} \rightarrow 0$ both integrals in (5) sum up to the following expression for the standard fundamental solution of the Laplace operator:

$$
\begin{aligned}
& E_{0}\left(y_{1}, y_{2}\right)=\frac{1}{4 \pi} \log \left(y_{1}^{2}+y_{2}^{2}\right) \\
& \quad=-\frac{1}{4 \pi} \operatorname{reg} \int_{0}^{\infty} \frac{\exp \left\{-\frac{1+i}{2} y_{1} \varrho-\frac{1-i}{2} y_{2} \varrho\right\}+\exp \left\{-\frac{1-i}{2} y_{1} \varrho-\frac{1+i}{2} y_{2} \varrho\right\}}{\varrho} d \varrho .
\end{aligned}
$$


The formula (5) can be geometrically interpreted as follows. Take the intersection points of Char $P$ with $\stackrel{\circ}{\alpha}+i \mathbb{R}^{2}$. If $\stackrel{\circ}{\alpha} \neq 0$ we get two points $\left(\stackrel{\circ}{\alpha}_{1} \pm i \stackrel{\circ}{\alpha}_{2}, \stackrel{\circ}{\alpha}_{2} \mp i \stackrel{\circ}{\alpha}_{1}\right)$. Next we take half-lines starting from those points, contained in the characteristic set and such that the real parts of points of those half-lines tend to infinity as their norms do. Then (5) represents the integral over such half-lines of the function which is the inverse of the distance over $\zeta$ between the two sheets of the characteristic variety.

This geometrical interpretation holds for a broad class of partial differental operators in two variables (see [Z2], Theorem 17.3).

EXAMPLE 2. For the standard solution of the Laplace operator in three dimensions we obtain the representation (valid for $y_{1}, y_{2}, y_{3}>0$ )

$$
\begin{aligned}
& E_{0}\left(y_{1}, y_{2}, y_{3}\right)=\left(y_{1}^{2}+y_{2}^{2}+y_{3}^{2}\right)^{-1 / 2} \\
& =\sum_{\varepsilon \in\{-,+\}^{2}} \operatorname{reg} \int_{0}^{\infty} \int_{0}^{\infty}\left(\gamma_{2}^{2}-\varepsilon_{1} \varepsilon_{2}\left(1+\varepsilon_{1} i\right) \gamma_{2} \gamma_{1}\right)^{-1 / 2} \cdot \exp \left\{-y_{1} \frac{1+\varepsilon_{1} i}{2} \gamma_{1}\right. \\
& \left.\quad+\varepsilon_{2} y_{2} \sqrt{\gamma_{2}^{2}-\varepsilon_{1} \varepsilon_{2}\left(1+\varepsilon_{1} i\right) \gamma_{2} \gamma_{1}}-y_{3}\left(\frac{1-\varepsilon_{1} i}{2} \gamma_{1}+\varepsilon_{2} i \gamma_{2}\right)\right\} d \gamma_{1} d \gamma_{2} .
\end{aligned}
$$

In the above integrals the branch of the square root function is chosen in such a way that the real parts of the exponents are positive.

\section{References}

[B] B. L. J. Braaksma, Multisummability of formal power series solutions of nonlinear meromorphic differential equations, Ann. Inst. Fourier (Grenoble) 42 (1992), $517-540$.

[Ec] J. Ecalle, Les fonctions résurgentes, Publ. Math. Univ. de Paris-Sud, 1980.

[Eh] L. Ehrenpreis, A fundamental principle for systems of linear differential equations with constant coefficients and some of its applications, in: Proc. Internat. Sympos. on Linear Spaces (Jerusalem, 1960), 1961, 161-174.

[L] J. Leray, Le calcul différentiel et intégral sur une variété analytique complexe, Bull. Soc. Math. France 87 (1959), 81-180.

[NZ] Nguyen Si Minh and B. Ziemian, A remark on Nilsson type integrals, in: Singularities and Differential Equations, Banach Center Publ. 33, Inst. Math., Polish Acad. Sci., Warszawa, 1996, 227-285.

[N] N. Nilsson, Some growth and ramification properties of certain multiple integrals, Ark. Mat. 5 (1965), 463-476.

[P] V. P. Palamodov, General form of solutions of linear differential operators with constant coefficients, Dokl. Akad. Nauk SSSR 143 (1962), 1278-1281 (in Russian).

[SZ] Z. Szmydt and B. Ziemian, The Mellin Transformation and Fuchsian Type Partial Differential Equations, Math. Appl. 56, Kluwer, 1992.

[Z1] B. Ziemian, Leray residue formula and asymptotics of solutions to constant coefficient PDEs, Topol. Methods Nonlinear Anal. 3 (1994), 257-293. 
[Z2] B. Ziemian, Generalized analytic functions with applications to singular ordinary and partial differential equations, Dissertationes Math. 354 (1996).

Institute of Mathematics

Polish Academy of Sciences

P.O. Box 137

00-950 Warszawa, Poland

E-mail: lysik@impan.gov.pl

Reçu par la Rédaction le 20.2.2000 